\title{
供体-锯齿型碳纳米管-受体的非线性光学响应对管径的依赖性
}

\author{
刘英涛* 王 金金刘翔宇冀永强
}

(宁夏大学化学化工学院 银川 750021)

\begin{abstract}
摘要 用密度泛函 B3LYP 方法得到一系列不同管径的氨基-硝基双取代纳米管 $\mathrm{NH}_{2}-(n, 0) \mathrm{CNT}^{-\mathrm{NO}_{2}}(n=5 \sim 10)$ 的几何 结构. 用 CAM-B3LYP 方法计算了体系的第一超极化率 $\left(\beta_{0}\right)$. 研究表明, $\beta_{0}$ 对管径大小有着极强的依赖性. 特别是对偶 数纳米管 $(n=6,8$ 和 10$)$, 其 $\beta_{0}\left(5.5 \times 10^{3} \sim 9.8 \times 10^{4} \mathrm{au}\right)$ 是奇数纳米管 $\beta_{0}\left(1.0 \times 10^{3} \sim 2.8 \times 10^{3} \mathrm{au}\right)$ 的 $5 \sim 35$ 倍.

关键词 碳纳米管; 非线性光学; 依赖性
\end{abstract}

\section{Nonlinear Optical Response in Donor-Zigzag Carbon Nanotube-Acceptor Dependence on the Tube Diameter}

\author{
Liu, Yingtao* Wang, Xin Liu, Xiangyu Ji, Yongqiang \\ (Department of Chemistry and Chemical Engineering, Ningxia University, Yinchuan 750021, China)
}

\begin{abstract}
A series of $\mathrm{NH}_{2}$ and $\mathrm{NO}_{2}$ disubstituted carbon nanotube $\mathrm{NH}_{2}-(n, 0) \mathrm{CNT}-\mathrm{NO}_{2}(n=5 \sim 10)$ were obtained by the DFT-B3LYP method. First hyperpolarizabilities $\left(\beta_{0}\right)$ were calculated at the CAM-B3LYP level. As a result, it is found that the first hyperpolarizability strongly depends on the diameter of CNT. Particularly, $\beta_{0}\left(5.5 \times 10^{3} \sim 9.8 \times 10^{4}\right)$ in the even CNT $(n=6,8$ and 10) is about $5 \sim 35$ times larger than that $\left(1.0 \times 10^{3} \sim 2.8 \times 10^{3}\right.$ au) in the odd CNT $(n=5,7$ and 9$)$.
\end{abstract}

Keywords carbon nanotube; nonlinear optics; dependence

近 20 年来, 人们一直致力于研究不同类型品质优 良的非线性光学物质 ${ }^{[1 \sim 0]}$. 供体(Donor)- $\pi$ 共轭桥-受体 (Acceptor) (D- $\pi-\mathrm{A}$ )框架已经成为设计高性能有机非线 性光学材料的成功范例 ${ }^{[1123]}$. 人们采用这种方案来设 计 NLO 材料时, 往往是增加共轭桥长或者改变推拉电 子对的强度来增加材料的 NLO 性质. 在以往的研究中, 多烯烃、苯、苯同系物以及吓啉环等 $\pi$ 共轭体系常被选 作 D- $\pi$-A 框架中的共轭桥来设计有机 NLO 材料. 最 近, 杨伟涛等 ${ }^{[24]}$ 采用碳纳米管 (CNT) 为共轭桥, 研究取 代碳纳米管的 NLO 性质以及共轭桥长度对 NLO 的影 响. 在本文中, 我们研究了管径(奇数管径和偶数管径) 对双取代碳纳米管第一超极化率的影响.

\section{1 计算方法}

使用 B3LYP/6-31G*方法优化了不同管径的氨基硝 基取代碳纳米管 $\mathrm{NH}_{2}-(n, 0) \mathrm{CNT}-\mathrm{NO}_{2}(n=5 \sim 10)$ 的几何 结构. 使用有限场的方法来计算体系的电学性质. 对于 第一超极化率的计算, 选择适当的计算方法是非常重要 的. 据报道 B3LYP 方法过高地估计了大的 D- $\pi$-A 体系 的第一超极化率 ${ }^{[25]}$. MP2 方法对超极化率的计算比 B3LYP 方法更为可靠, 但对于大体系来说, 采用这种方 法的计算成本又非常高。一种新的密度泛函 CAM-B3LYP 方法 ${ }^{[26,27]}$ 被发展并被验证能够很好地预测 大的电荷转移体系 ${ }^{[28 ~ 30]}$ 的分子性质. 我们选择了一个

\footnotetext{
* E-mail: liuyingtao2004@126.com

Received November 29, 2011; accepted January 20, 2012.
}

Supported by the Natural Science Foundation of Ningxia Province (No. NZ1105) and the Scientific Research Fund of Ningxia University (No. NDZR10-14).

宁夏自然科学基金(No. NZ1105)联合资助项目, 宁夏大学科学研究基金(No. NDZR10-14)重点资助项目. 
中等尺度的 $\mathrm{H}_{2} \mathrm{~N}-(6,0) \mathrm{CNT}-\mathrm{NO}_{2}$ 体系 (见图 1) 来对 CAM-B3LYP 方法的可靠性进行测试. 对同一体系我们 采用了不同的计算方法, 如 HF, LC-BLYP, BhandHLYP, CAM-B3LYP 和 MP2 来计算它的第一超极化率，结果列 于表 1. 从表中, 我们可以看出 CAM-B3LYP 对第一超 极化率的结果更接近 MP2 的结果, 误差仅为 $4.6 \%$. 因 此, 在以下的计算中, 我们采用了 CAM-B3LYP 方法来 计算第一超极化率. 对所有原子采用 6-31+G(d)基组. 选用高斯的默认电场 $0.001 \mathrm{au}$ 作为体系的应用电场. 这 个电场值已经被应用在很多第一超极化率的计算 中 $^{[10,21 \sim 23]}$.

表 1 HF, LC-BLYP, BHandHLYP, CAM-B3LYP 和 MP2 水平 下 $\mathrm{NH}_{2}-(6,0) \mathrm{CNT}-\mathrm{NO}_{2}$ 的静态第一超极化率

Table 1 Static first hyperpolarizability $\left(\beta_{0}\right)$ at the different levels, such as HF, LC-BLYP, BHandHLYP, CAM-B3LYP, and MP2, for the medium sized $\mathrm{NH}_{2}-(6,0) \mathrm{CNT}-\mathrm{NO}_{2}$ system

\begin{tabular}{ccc}
\hline Method & $\beta_{0}$ & Error/\% \\
\hline HF & 1550 & 73.1 \\
LC-BLYP & 2266 & 60.7 \\
BHandHLYP & 5473 & 5.0 \\
CAM-B3LYP & 5497 & 4.6 \\
MP2 & 5760 & 0.0 \\
\hline
\end{tabular}

分子在均匀的外加电场中, 分子的能量是场强的函 数. 分子在弱场下, 它的能量可以用以下公式表示:

$$
E=E^{0}-\mu_{\alpha} F_{\alpha}-\frac{1}{2} \alpha_{\alpha \beta} F_{\alpha} F_{\beta}-\frac{1}{6} \beta_{\alpha \beta \gamma} F_{\alpha} F_{\beta} F_{\gamma} \cdots
$$

在公式中, 在没有施加外加电场时体系的能量为 $E^{0}, F_{\alpha}$ 表示外加电场在 $\alpha$ 方向上的一个分量. $\mu_{\alpha}, \alpha_{\alpha \beta}$ 和 $\beta_{\alpha \beta \gamma}$ 分别表示偶极距、极化率和第一超极化率的一个分 量. 我们主要研究其极化率 $\alpha_{0}$ 和第一超极化率 $\beta_{0}$. 偶极距和极化率定义如下:

$$
\mu_{0}=\left(\mu_{x}^{2}+\mu_{y}^{2}+\mu_{z}^{2}\right)^{1 / 2}, \quad \alpha_{0}=\frac{1}{3}\left(\alpha_{x x}+\alpha_{y y}+\alpha_{z z}\right)
$$

静态第一超极化率定义如下:

$$
\beta_{0}=\left(\beta_{x}^{2}+\beta_{y}^{2}+\beta_{z}^{2}\right)^{1 / 2}
$$

其中 $\beta_{x}=\frac{3}{5}\left(\beta_{x x x}+\beta_{x y y}+\beta_{x z z}\right), \quad \beta_{y}=\frac{3}{5}\left(\beta_{y y y}+\beta_{y x x}+\beta_{y z z}\right)$, $\beta_{z}=\frac{3}{5}\left(\beta_{z z z}+\beta_{z x x}+\beta_{z y y}\right)$. 所有计算均使用 Gaussian 09 程 序完成 ${ }^{[31]}$.

\section{2 结果与讨论}

\section{1 平衡几何}

图 1 显示了不同管径的锯齿型氨基-硝基 $\left(\mathrm{NH}_{2}-\mathrm{NO}_{2}\right)$ 双取代碳纳米管 $\mathrm{NH}_{2}-(n, 0) \mathrm{CNT}-\mathrm{NO}_{2}(n=5 \sim 10)$ 的几何 构型, 其结构是在 B3LYP/6-31G*水平下得到的, 我们 所研究的纳米管被称为超短纳米管 ${ }^{[32,33]}$. 为了便于讨论 管径对体系 NLO 性质的影响, 我们将硝基和氨基以面 对面方式取代在纳米管两端(如图 1 所示), 这里我们不 讨论取代位置对 NLO 的影响.
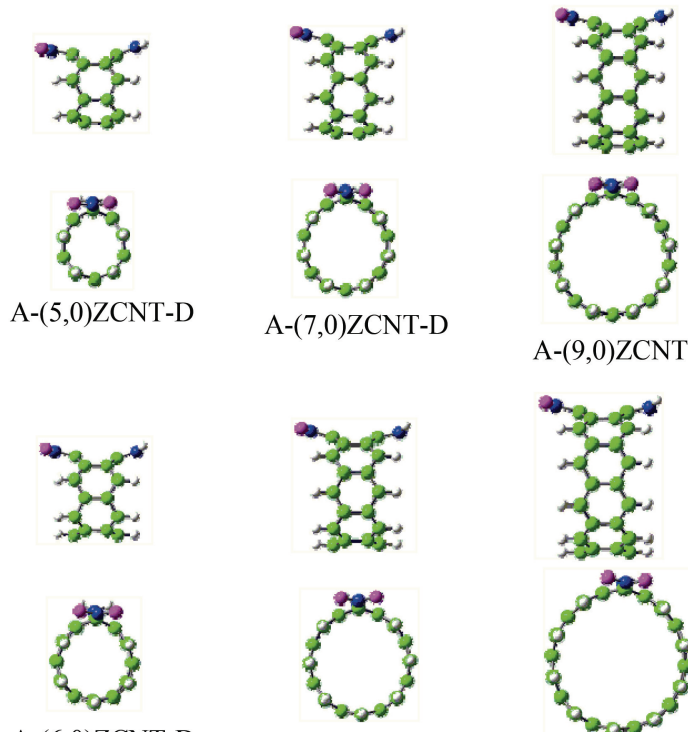
A- $(9,0)$ ZCNT-D

A- $(6,0)$ ZCNT-D
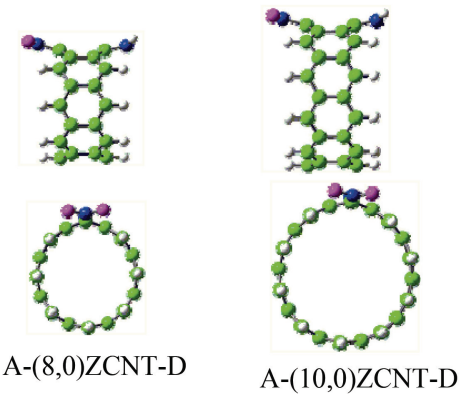

图 $1 \mathrm{D}-(n, 0)$ CNT-A $(n=5 \sim 10)\left(\mathrm{D}=\mathrm{NH}_{2}\right.$ and $\left.\mathrm{A}=\mathrm{NO}_{2}\right)$ 的优化 结构. 绿、蓝、粉色以及白色小球分别代表 $\mathrm{C}, \mathrm{N}, \mathrm{O}$ 和 $\mathrm{H}$ 原子 Figure 1 The optimized structures of D- $(n, 0) \mathrm{CNT}-\mathrm{A}(n=5 \sim$ 10) $\left(\mathrm{D}=\mathrm{NH}_{2}\right.$ and $\left.\mathrm{A}=\mathrm{NO}_{2}\right)$. The green, blue, pink and white balls denote $\mathrm{C}, \mathrm{N}, \mathrm{O}$ and $\mathrm{H}$ atoms, respectively

\section{2 非线性光学性质}

在 CAM-B3LYP/6-31 + G(d) 水平下, 计算了 $\mathrm{NH}_{2}-(n, 0)$ CNT- $\mathrm{NO}_{2}(n=5 \sim 10)$ 的极化率 $\left(\alpha_{0}\right)$ 和第一超极 化率 $\left(\beta_{0}\right)$. 从表 2 中我们得知, 当 $n$ 等于奇数 5,7 和 9 时, $\mathrm{NH}_{2}-(n, 0)$ CNT- $\mathrm{NO}_{2}$ 的 $\alpha_{0}$ 值缓慢增加, 从 $267(n=5)$ 增加 到 $538 \mathrm{au}(n=9)$. 同样它们 $\beta_{0}$ 值的增加也不是很明显, 从 $1.0 \times 10^{3}(n=5)$ 增加到 $2.8 \times 10^{3} \mathrm{au}(n=9)$, 增幅不到 3 倍. 当 $n$ 等于偶数 6,8 和 10 时, $\mathrm{NH}_{2}-(n, 0) \mathrm{CNT}-\mathrm{NO}_{2}$ 的 $\alpha_{0}$ 值从 $338(n=6)$ 增加到 $775(n=10)$, 增幅和 $n$ 等于奇数 体系时相差不多. 与奇数体系有所不同的是, 偶数体系 的 $\beta_{0}$ 值迅速增加, 从 $5.5 \times 10^{3}(n=6)$ 增加到 $9.8 \times 10^{4} \mathrm{au}$ $(n=10)$, 增幅近 18 倍. 为了能够看的更为直观, 我们将 $\beta_{0}$ 值的变化展示在图 2 中. 偶数体系的 $\beta_{0}\left(5.5 \times 10^{3} \sim\right.$ $\left.9.8 \times 10^{4} \mathrm{au}\right)$ 明显高于奇数的 $\beta_{0}\left(1.0 \times 10^{3} \sim 2.8 \times 10^{3} \mathrm{au}\right)$. 
表 2 跃迁能 $\Delta E(\mathrm{eV})$, 振子强度 $f_{0}$, 极化率 $\alpha_{0}(\mathrm{au})$ 和第一极化 率 $\beta_{0}(\mathrm{au})$

Table 2 Transition energy $\Delta E(\mathrm{eV})$, oscillator strength $f_{0}$, polarizability $\alpha_{0}(\mathrm{au})$, and first hyperpolarizability $\beta_{0}(\mathrm{au})$

\begin{tabular}{lcccc}
\hline Molecule & $\Delta E$ & $f_{0}$ & $\alpha_{0}$ & $\beta_{0}$ \\
\hline $\mathrm{NH}_{2}-(5,0) \mathrm{CNT}-\mathrm{NO}_{2}$ & 3.91 & 0.098 & 268 & $1.0 \times 10^{3}$ \\
$\mathrm{NH}_{2}-(7,0) \mathrm{CNT}-\mathrm{NO}_{2}$ & 3.53 & 0.071 & 387 & $1.8 \times 10^{3}$ \\
$\mathrm{NH}_{2}-(9,0) \mathrm{CNT}-\mathrm{NO}_{2}$ & 2.86 & 0.094 & 538 & $2.8 \times 10^{3}$ \\
$\mathrm{NH}_{2}-(6,0) \mathrm{CNT}-\mathrm{NO}_{2}$ & 2.62 & 0.199 & 338 & $5.5 \times 10^{3}$ \\
$\mathrm{NH}_{2}-(8,0) \mathrm{CNT}-\mathrm{NO}_{2}$ & 2.20 & 0.424 & 550 & $2.4 \times 10^{4}$ \\
$\mathrm{NH}_{2}-(10,0) \mathrm{CNT}-\mathrm{NO}_{2}$ & 1.64 & 0.189 & 775 & $9.8 \times 10^{4}$ \\
\hline
\end{tabular}

为什么在 $\mathrm{NH}_{2}-(n, 0) \mathrm{CNT}-\mathrm{NO}_{2}$ 体系中, $n$ 等于偶数体 系的 $\beta_{0}$ 值要远高于 $n$ 等于奇数体系的 $\beta_{0}$ 值呢? 为了探 明上述现象的本质, 我们采用 Oudar 等 ${ }^{[34]}$ 提出的双能级 公式对其进行解释.

$$
\beta_{0} \propto \frac{\Delta \mu \cdot f_{0}}{\Delta E^{3}}
$$

式中, $\Delta E 、 f_{0}$ 和 $\Delta \mu$ 分别代表跃迁能、振子强度和基态与 激发态之间的偶极距差. 从公式中, 我们可以看出, $\Delta E$ 的 3 次方反比于 $\beta_{0}$, 因此 $\Delta E$ 是影响 $\beta_{0}$ 变化的主要因素. 由表 2 可见, 当 $n$ 分别等于奇数 5,7 和 9 时, 它们的振 子强度相近 $(0.071 \sim 0.098)$, 其 $\beta_{0}$ 值随着 $\Delta E$ [3.91 $(n=$ $5), 3.53(n=7)$ 和 $2.86 \mathrm{eV}(n=9)]$ 减小而增加. 当 $n$ 分别 等于偶数 6,8 和 10 时, 它们的振子强度 $(0.189 \sim 0.424)$ 同样相近, 其 $\beta_{0}$ 值随着 $\Delta E$ [2.62 $(n=6), 2.20(n=8)$ 和 $1.64 \mathrm{eV}(n=10)]$ 减小而增加. 无论对于奇数体系还是偶 数体系, 它们 $\beta_{0}$ 值的变化都很好地满足了双能级公式.

下面我们比较奇数体系和偶数体系的振子强度和 跃迁能, 发现偶数体系的振子强度 $(0.189 \sim 0.424)$ 明显 大于奇数体系的振子强度 $(0.071 \sim 0.098)$. 同时偶数体 系的跃迁能(1.64 2.62 eV) 明显小于奇数体系的跃迁能 $(2.86 \sim 3.91 \mathrm{eV})$. 因此 $\beta_{0}$ 值出现了图 2 所示的变化趋势, 偶数碳管的 $\beta_{0}$ 值远高于奇数碳管的 $\beta_{0}$ 值. 为了更好地理 解奇偶纳米管对 $\beta_{0}$ 的影响, 我们单独来研究一下共轭桥 $(n, 0) \mathrm{CNT}(n=5 \sim 10)$ 本身的性质, 图 3 表示的是 $(n, 0) \mathrm{CNT}$ 的最高占据分子轨道( $\mathrm{HOMO})$ 和最低非占据轨 道(LUMO)以及能隙(HOMO-LUMO). 从图 3 可以清晰 看到, 由于 $n$ 的奇偶性即纳米管对称性的不同, 纳米管 的 HOMO 与 LUMO 轨道呈现出两类不同的形状, 偶数 纳米管的 HOMO 是苯环相对碳原子上的轨道发生离域, 而奇数纳米管的 HOMO 是苯环相邻的碳原子轨道发生 离域. 从能隙上看, 偶数纳米管能隙也小于奇数纳米管 的能隙, 能隙越小, 越利于电子跃迁, 能隙的顺序与上 述跃迁能的顺序是一致的.

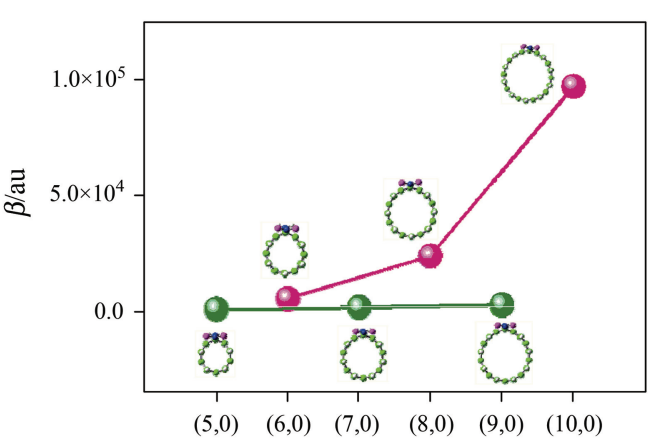

图 2 在 $\mathrm{NH}_{2}-(n, 0) \mathrm{CNT}-\mathrm{NO}_{2}$ 中, 第一超极化率和 $n$ 的关系

Figure 2 Relationship between the first hyperpolarizability and $n$ in $\mathrm{NH}_{2}-(n, 0) \mathrm{CNT}-\mathrm{NO}_{2}$

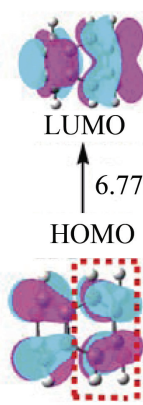

$(5,0) \mathrm{CNT}$

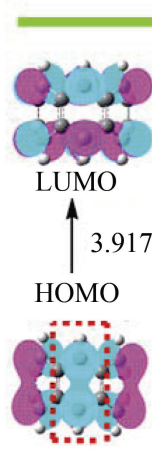

$(6,0) \mathrm{CNT}$
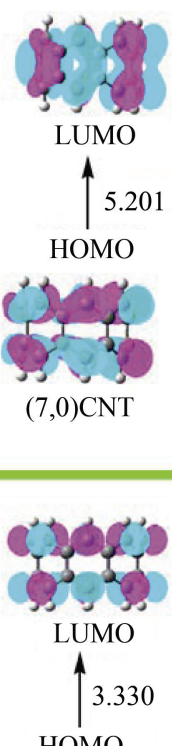

$\mathrm{HOMO}$

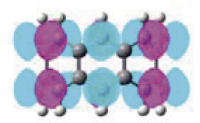

$(8,0) \mathrm{CNT}$

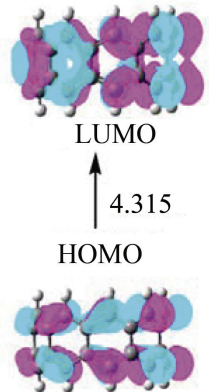

$(9,0) \mathrm{CNT}$
图 3 在 $\mathrm{HF} / 6-31 \mathrm{G}^{*} / / \mathrm{B} 3 \mathrm{LYP} / 6-31 \mathrm{G}^{*}$ 水平下, $(n, 0) \mathrm{CNT}(n=5 \sim$ $10)$ 的最高占据轨道、最低非占据轨道以及能隙 $(\mathrm{eV})$

Figure 3 HOMO, LUMO and energy gap $(\mathrm{eV})$ of $(n, 0) \mathrm{CNT}$ $(n=5 \sim 10)$ at the HF/6-31G*//B3LYP/6-31G* level

\section{3 结论}

综上所述，本文利用密度泛函理论研究了双取代 锯齿型碳纳米管管径变化与体系非线性光学性质的依 赖关系, 发现在 $\mathrm{NH}_{2}-(n, 0) \mathrm{CNT}-\mathrm{NO}_{2}$ 体系中, $n$ 为偶数时 体系的 $\beta_{0}$ 值明显高于 $n$ 为奇数的 $\beta_{0}$ 值, $n$ 为偶数的纳米 管有利于非线性光学材料的设计, 此研究结果将对合成 和设计新型 NLO 材料提供理论帮助. 


\section{References}

1 Eaton, D. F. Science 1991, 253, 281.

2 Meredith, G. R. Nonlinear Optical Properties of Organic and Polymeric Materials, ACS Symposium Series 233, Ed.: Williams, D. J., American Chemical Society, Washington, 1983.

3 Cheng, W. D.; Xiang, K. H.; Pandey, R.; Pernisz, U. C. J. Phys. Chem. B 2000, 104, 6737.

4 Ichida, M.; Sohda, T.; Nakamura, A. J. Phys. Chem. B 2000, 104, 7082 .

5 Hold, K.; Pawlowski, F.; Jørgensen, P.; Hättig, C. J. Chem. Phys. 2003, 118, 1292.

6 Hättig, C.; Larsen, H.; Olsen, J.; Jørgensen, P.; Koch, H.; Femández, B.; Rizzo, A. J. Chem. Phys. 1999, 111, 10099.

7 Horikoshi, R.; Nambu, C.; Mochida, T. Inorg. Chem. 2003, $42,6868$.

8 Long, N. J.; Williams, C. K. Angew. Chem., Int. Ed. 2003, 42, 2586.

9 Shelton, D. P.; Rice, J. E. Chem. Rev. 1994, 94, 3.

10 Wu, W. B.; Wang, M. L.; Huang, W.; Sun, Y. M.; Cui, Y. P.; Xu, C. X. Acta Chim. Sinica 2008, 66, 1700 (in Chinese). (吴伟兵, 王明亮, 黄维, 孙岳明, 崔一平, 徐春祥, 化学 学报, 2008, 66, 1700.)

11 Blanchard-Desce, M.; Alain, V.; Bedworth, P. V.; Marder, S. R.; Fort, A.; Runser, C.; Barzoukas, M.; Lebus, S.; Wortmann, R. Chem.-Eur. J. 1997, 3, 1091.

12 Champagne, B.; Perpète, E. A.; Jacquemin, D.; Jacquemin, D. J. Phys. Chem. A 2000, 104, 4755.

13 Nakano, M.; Ohta, S.; Kamada, K.; Kishi, R.; Kubo, T.; Kamada, K.; Ohta, K.; Champagne, B.; Botek, E.; Takahashi, H. Chem. Phys. Lett. 2007, 443, 95.

14 Kanis, D. R.; Ratner, M. A.; Marks, T. J. Chem. Rev. 1994, 94, 195.

15 Priyadarshy, S.; Therien, M. J.; Beratan, D. N. J. Am. Chem. Soc. 1996, 118, 1504.

16 Suslick, K. S.; Chen, C. T.; Meredith, G. R.; Cheng, L. T. J. Am. Chem. Soc. 1992, 114, 6928.

17 Marder, S. R.; Beratan, D. N.; Cheng, L.-T. Science 1991, 252, 103.

18 Priyadarshy, S.; Therien, M. J.; Beratan, D. N. J. Am. Chem. Soc. 1996, 118, 1504.

19 Bishop, D. M.; Champagne, B.; Kirtman, B. J. Chem. Phys. 1998, 109, 9987.

20 Bulat, F. A.; Toro-Labbe, A.; Champagne, B.; Kirtman, B.; Yang, W. J. Chem. Phys. 2005, 123, 014309.

21 Zhou, Z. J.; Li, X. P.; Liu, Z. B.; Ma, F.; Li, Z. R.; Huang,
X. R.; Sun, C. C. Chem.-Eur. J. 2011, 17, 2414.

22 Zhou, Z. J.; Liu, Z. B.; Li, Z. R.; Huang, X. R.; Sun, C. C. J. Phys. Chem. C 2011, 115, 16282.

23 Zhou, Z. J.; Li, Z. R.; Huang, X. R.; Sun, C. C. Chem. Res. Chin. Univ. 2011, 27(3), 512.

24 Xiao, D.; Bulat, F. A.; Yang, W.; Beratan, D. N. Nano Lett. 2008, 8, 2814

25 Champagne, B.; Perpète, E.; Jacquemin, D.; Van Gisbergen, S.; Baerends, E.; Soubra-Ghaoui, C.; Robins, K.; Kirtman, B. J. Phys. Chem. A 2000, 104, 4755.

26 Nakano, M.; Ohta, S.; Kamada, K.; Kishi, R.; Kubo, T.; Kamada, K.; Ohta, K.; Champagne, B.; Botek, E.; Takahashi, H. Chem. Phys. Lett. 2007, 443, 95.

27 Nakano, M.; Nagai, H.; Fukui, H.; Yoneda, K.; Kishi, R.; Takahashi, H.; Shimizu, A., Kubo, T.; Kamada, K.; Ohta, K. Chem. Phys. Lett. 2008, 467, 120.

28 Nagai, H.; Nakano, M.; Yoneda, K.; Fukui, H.; Minami, T.; Boness, S.; Kishi, R.; Takahashi, H.; Shimizu, A.; Kubo, T.; Kamada, K.; Ohta, K. Chem. Phys. Lett. 2009, 477, 355.

29 Ma, F.; Li, Z.; Zhou, Z.; Wu, D.; Li, Y.; Wang, Y.; Li, Z. J. Phys. Chem. C 2010, 114, 11242.

30 Tawada, Y.; Tsuneda, T.; Yanagisawa, S.; Yanai, T.; Hirao, K. J. Chem. Phys. 2004, 120, 8425.

31 Frisch, M. J.; Truchks, G. W.; Schlegel, H. B.; Scuseria, G. E.; Robb, M. A.; Cheeseman, J. R.; Scalmani, G.; Barone, V.; Mennucci, B.; Petersson, G. A.; Nakatsuji, H.; Caricato, M.; Li, X.; Hratchian, H. P.; Izmaylov, A. F.; Bloino, J.; Zheng, G.; Sonnenberg, J. L.; Hada, M.; Ehara, M.; Toyota, K.; Fukuda, R.; Hasegawa, J.; Ishida, M.; Nakajima, T.; Honda, Y.; Kitao, O.; Nakai, H.; Vreven, T.; Montgomery, Jr., J. A.; Peralta, J. E.; Ogliaro, F.; Bearpark, M.; Heyd, J. J.; Brothers, E.; Kudin, K. N.; Staroverov, V. N.; Kobayashi, R.; Normand, J.; Raghavachari, K.; Rendell, A.; Burant, J. C.; Iyengar, S. S.; Tomasi, J.; Cossi, M.; Rega, N.; Millam, N. J.; Klene, M.; Knox, J. E.; Cross, J. B.; Bakken, V.; Adamo, C.; Jaramillo, J.; Gomperts, R.; Stratmann, R. E.; Yazyev, O.; Austin, A. J.; Cammi, R.; Pomelli, C.; Ochterski, J. W.; Martin, R. L.; Morokuma, K.; Zakrzewski, V. G.; Voth, G. A.; Salvador, P.; Dannenberg, J. J.; Dapprich, S.; Daniels, A. D.; Farkas, Ö.; Foresman, J. B.; Ortiz, J. V.; Cioslowski, J.; Fox, D. J. Gaussian 09, Revision A.02, Gaussian, Wallingford, CT, 2009.

32 Xu, H. L.; Zhong, R. L.; Sun, S. L.; Su, Z. M. J. Phys. Chem. C 2011, 115, 16340.

33 Sun, C. H.; Finnerty, J. J.; Lu, G. Q.; Cheng, H. M. J. Phys. Chem. B 2005, 109, 12406.

34 Oudar, J. L.; Chemla, D. S. J. Chem. Phys. 1977, 66, 2664. 